\title{
Motion Analysis of Hammer Throw Technique of the World Top Female Athletes
}

\author{
Liqiang Li ${ }^{1, \mathrm{a}}$, Bingjun Wan ${ }^{1, \mathrm{~b}}$, Cheng Guo ${ }^{1, \mathrm{c}}$, Yuliang Sun ${ }^{1, \mathrm{~d}}$ \\ ${ }^{1}$ School of Physical Education, Shaanxi Normal University, Shaanxi 710119, China; \\ allq192104@163.com, bbingjunw55@snnu.edu.cn, '1017325605@qq.com, dsunsyl123@163.com
}

Keywords: hammer throw; kinematic analysis; female athlete

\begin{abstract}
In order to study the characteristics of the best Chinese female athlete Zheng Wang's hammer throw technique and improve her performance, we used biomechanical video analysis to analyze the technical characteristics of 6 world top female hammer players. We found that in the process of holding the ball, Zheng Wang's double stance and single stance phases in the first, third and fourth turn was reasonable, but in the second turn it was unbalanced. (2) Zheng Wang's rotation were relatively faster, and rotation was fluent. She was one of the fastest female hammer throwers in the world. (3)During the rotation in the last turn, Zheng Wang maintained the same rotation time as the third circle, which lead to less speed-up, compare with the third circle.(4) During the rotation, the Angle of the fan was basically reasonable, but the increment of the second, third and fourth circle was unstable than that of the previous turns. (5)Through the long-term observation of Zheng Wang's training and competition, it was found that there were some problems in the psychological stability of the competition.
\end{abstract}

\section{Introduction}

Since enrolled in the world athletics championships in 1999 and the Olympic Games in Sydney in 2000, women's hammer project has been one of the biggest concerns in the long throwing events. Hammer is also one of the most complicated and exciting events in track and field events.

Top six female hammer players in the 2017 London world championships: Anita Wlodarczyk, Malwina Kopron, Zheng Wang, Wenxiu Zhang, Hanna Skydan, Joanna Flodorow were taken as subjects, and Chinese hammer player Zheng Wang was the key point. Analyze the data of the athletes' presets, rotation of each circle, supporting time of single-foot and double-foot, etc. To study the throwing processes and technical characteristics of the players.

\section{Methods}

Firstly, we reviewed a large number of literature relate to women hammer throwing skills analysis in order to provide theoretical support. Secondly, video clips were used to select the video clips we need. We also had the female hammer videos of the 2017 London track and field world championships .By using the professional video analysis system, we analyzed the technical characteristics of the athletes and get the conclusions.

The main subject this study is Zheng Wang, a top sportswoman at international level from Shaanxi province of China. She was born on December 14, 1987. On August 8, 2017, Zheng Wang won a silver medal in the female hammer in the world championships in London. She is one of the key athletes of China's track and field team preparing for the 2020 Tokyo Olympic Games.

\section{Results}

Hammer technique is often divided into three major technical phases: preliminary swings, rotation and final exertion in teaching and researches. Athletes apply an initial force to the apparatus, so that the devices have initial velocities in the preliminary swings procedure. Rotation technology is the core part of athletes applying actual force to the apparatus, and usually top athletes in the world adopt 
four-circle rotation technology. Each rotation of the four circles was interchanged by single-foot and double stance phase respectively. Before throwing the hammer, athletes should try their best to surpass the device's action effect by technical motion and personal physical characteristics, so as to reach a better effect of imposing the power generate from motion to hammers. Relevant studies have also shown that: prolong double stance phase time and shorten single stance phasing time was the development trend of hammer in the future, and it was also the direct expression of the modern hammer throwing technology [1].

\subsection{Analysis of the rotation time of the top six female hammer players in the World Championships in London}

The top six female hammer players in the 2017 world championships in London all adopted four-circle rotation technology. They used the rotation technique to complete each circle with single-foot and double stance phase, and speed up the swing through the interchange and adjust supporting. The table below is analysis of the top six female hammer players' single-foot and double stance phase time in the World Championships in London.

Table 1Single-foot and double stance phase time of each circle

in the top six female hammer players in World Championships in London (s)

\begin{tabular}{|c|c|c|c|c|c|c|c|c|c|c|}
\hline \multirow{2}{*}{ Place } & \multirow{2}{*}{ Name } & \multicolumn{2}{|c|}{ First turn } & \multicolumn{2}{|c|}{ Second turn } & \multicolumn{2}{|c|}{ Third Ring } & \multicolumn{2}{|c|}{ Fourth Ring } & \multirow{2}{*}{$\begin{array}{c}\mathrm{DS} / \mathrm{S} \\
\mathrm{S}\end{array}$} \\
\hline & & DS & SS & DS & SS & DS & SS & DS & SS & \\
\hline 1 & $\begin{array}{c}\text { Anita } \\
\text { WLODARCZYK }\end{array}$ & 0.32 & 0.28 & 0.32 & 0.24 & 0.28 & 0.24 & 0.24 & 0.24 & 1.16 \\
\hline 2 & Zheng WANG & 0.32 & 0.32 & 0.32 & 0.20 & 0.24 & 0.20 & 0.22 & 0.22 & 1.17 \\
\hline 3 & $\begin{array}{l}\text { Malwina } \\
\text { KOPRON }\end{array}$ & 0.36 & 0.20 & 0.36 & 0.24 & 0.32 & 0.20 & 0.24 & 0.20 & 1.52 \\
\hline 4 & Wenxiu ZHANG & 0.32 & 0.28 & 0.28 & 0.24 & 0.28 & 0.24 & 0.24 & 0.28 & 1.08 \\
\hline 5 & Hanna SKYDAN & 0.32 & 0.28 & 0.36 & 0.24 & 0.32 & 0.24 & 0.24 & 0.22 & 1.27 \\
\hline 6 & $\begin{array}{c}\text { Joanna } \\
\text { FIODOROW }\end{array}$ & 0.36 & 0.24 & 0.32 & 0.20 & 0.30 & 0.24 & 0.24 & 0.24 & 1.33 \\
\hline $\mathrm{X}$ & & 0.33 & 0.27 & 0.33 & 0.23 & 0.29 & 0.23 & 0.24 & 0.23 & 1.26 \\
\hline
\end{tabular}

The above table shows that: in the acceleration phase of the first turn, all the six athletes' double-feet supporting time was in the mean of 0.33 seconds. All the supporting time ratios of single-foot and double-foot were greater than or equal to 1 , this means the supporting time ratios were reasonable. Relevant researches show that the rotation effect was the best when the double stance phase time accounts for 55\% of the total rotation time [2]. During the second circle of Zheng Wang's rotation, there was an imbalance in the supporting time of single-foot and double-foot, and double stance phase time increased from $50 \%$ in the first turn to $61.54 \%$. This float was consist with the data collected in the Chinese track and field grand prix (Zheng Zhou) and skill analysis. This fluctuation affects speed increments, acceleration increments, and angle of delivery. In the third and fourth turns, Zheng Wang's single-foot and double stance phase time was reasonable. In conclusion, during Zheng Wang's rotation process, the first, third and fourth turn supporting time distribution was reasonable. However, a slight imbalance occurred in single-foot and double stance phase during the rotation of the second circle, double stance phase time too long affects the acceleration process and final throwing effect [3].

The four-circle rotation was the process of speeding up hammers through athletes' rotation, and acquire bigger initial velocity throwing distance. There is obvious difference in rotation time in each turn of different athletes. Times should be gradually shortened from the first turn to fourth turn by acquiring acceleration through rotation. 


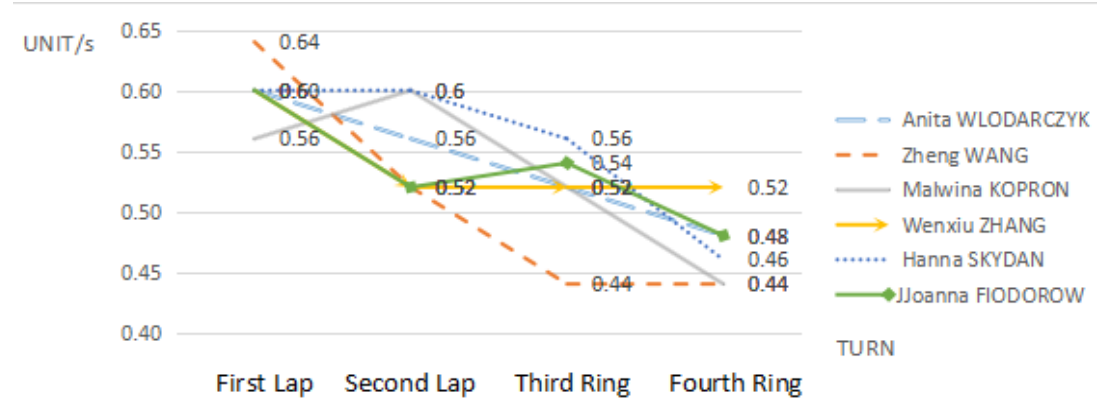

Fig. 2 Time of each rotation circle of top six female hammer players

in the World Championships in London

Fig. 2 shows that in the first turn, the longest rotation time was Zheng Wang's, about 0.64 seconds. Malwina Kopron took the shortest time of 0.56 seconds, the other athletes took 0.60 seconds. The average time of the six athletes in the first turn was 0.60 seconds. The longest time in the fourth turn was Wenxiu ZHANG, which was 0.52 seconds. The shortest was Zheng Wang and Malwina KOPRON, which lasted for 0.44 seconds. The average time of the six athletes in the fourth turn was 0.47 seconds. In this six athletes, only Anita Wlodarczyk had a linear change from the first to the fourth turn, the overall change trend was $0.60,0.56,0.52,0.48$ seconds, respectively. It shows that Anita WLODARCZYK has stable acceleration and fluent movements in all the four cycles. Therefore, she gained a better performance. The rotation times of Zheng Wang in the first three circles were also basically linear, which were $0.64,0.52,0.44$ seconds and the acceleration was obvious. While in the fourth turns, Zheng Wang's time was also 0.44 seconds, which was the same as that of the third circle, and the rotation speed was not increased considerably. Changes in speed during the rotation of other athletes also showed signs of instability. Analysis shows that the rotation time of Zheng Wang in the last turn was the same as that of the third circle, the rotation speed was similar. This was the technical adjustment to maintain stability, but it will affect the maximum speed of the final shot.

\subsection{Fan analysis of the rotation of top six female hammer athletes in the World Championships in London}

In the researches of hammer, the angle of the fan was used to reflect the ability of the players to overcome the centrifugal force caused by the device [4]. The angle of the fan was also called angle of the hammer's track, it was the angle between the line from the lowest point to the highest point and horizontal plane. In the process of acceleration, as the rotation speed of the athletes accelerates, the lowest point of the hammer should gradually decrease, and the highest point should gradually increase, thus the angle of the fan should be gradually larger. Angle of the fan was the main parameter to determine the ability of the athlete to overcome the centrifugal rate and the control ability of the device. The larger angle of the fan was, the stronger the ability to overcome the centrifugal rate of the athletes and the control ability of the apparatus would be. As the rotation speeds up and the speed of the hammer was increasing, the centrifugal effect of the apparatus was increasing. Thus athletes need to constantly change the position of body movement so that the angle of the trajectory of the instrument and the position of the lowest and highest points meet the requirements of the modern throwing technique. 
Table 3 Analysis of the top six female hammer athletes' angle of fan in the World Championships in London $\left(^{\circ}\right)$

\begin{tabular}{ccccccccc}
\hline \multirow{2}{*}{ Place } & \multirow{2}{*}{ Name } & $\begin{array}{c}\text { First } \\
\text { turn }\end{array}$ & $\begin{array}{c}\text { Secon } \\
\text { d turn }\end{array}$ & Increase & $\begin{array}{c}\text { Third } \\
\text { Ring }\end{array}$ & Increase & $\begin{array}{c}\text { Fourth } \\
\text { Ring }\end{array}$ & Increase \\
\hline 1 & Anita WLODARCZYK & 28 & 31 & 3 & 34 & 3 & 38 & 4 \\
2 & Zheng Wang & 27 & 31 & 4 & 34 & 3 & 39 & 5 \\
3 & Wenxiu Zhang & 31 & 33 & 2 & 36 & 3 & 40 & 4 \\
4 & Malwina KOPRON & 28 & 30 & 2 & 33 & 3 & 36 & 3 \\
5 & Hanna SKYDAN & 29 & 32 & 3 & 36 & 4 & 39 & 3 \\
6 & Joanna FLODOROW & 30 & 32 & 2 & 35 & 3 & 37 & 2 \\
\hline
\end{tabular}

According to statistics and analysis of the angle of fans in table 3, it can be seen that during the throwing process of the six athletes, the average angle of the fan from the first turn to the fourth circle increased from 28.8 degrees to 31.5 degrees, 34.7 degrees and 38.2 degrees respectively. Both Anita Wlodarczyk and Zheng Wang were close to the mean value. In other words, the average angle of fans of the first six athletes was close to Anita Wlodarczyk and Zheng Wang, and the comparative analysis showed that Zheng Wang and Anita Wlodarczyk had reasonable angles of rotation, and angles of delivery were moderate.

After analyzing the incremental changes in the angle of fan of the six athletes in the process of rotation, we can see that the champion, Anita Wlodarczyk, the angle increment was 4, 3 and 3 respectively, and the angle increment decreased in turn. In addition, Wenxiu Zhang and Malwina Kopron's angle increment in rotation also showed an increasing trend. It is shown that these athletes have good control ability in the process of rotation. In the rotation process of Zheng Wang, the angle increment was $5^{\circ}, 3^{\circ}$ and $4^{\circ}$, respectively, and the stability of angle increment is not strong lead to the weakness of Zheng Wang's control of the apparatus.

\section{Conclusions}

During Zheng Wang's rotation of hammer, the distribution of single-foot and double stance phase time in the first, third and fourth turns were reasonable, but that of the second rotation was unbalanced, the ratio increased to 1.6 , which was beyond $55 \%$ (double stance phase time should account for $55 \%$ of the total rotation time). This leads to instability of the "man-hammer" system and in turn affects the acceleration smoothness and final throwing effect.

Zheng Wang's time in the overall rotation and throwing process was relatively short, and had a good rotation fluency. She is one of the fastest female hammer players in the world to complete the whole throwing process. However, in the meantime of fast completion of action, perfect performance of the technical stability needs to be improved.

Zheng Wang maintained the same rotation time as the third turn during the rotation of the last turn, and acceleration was not obvious compared to the third circle. It is harmful for athletes to break through themselves and create better athletic performance.

Angle of fan in the rotation of Zheng Wang was basically reasonable, but the increment of the second circle, the third circle and the fourth circle was more unstable than that of the previous circle. The angle increment was "5-3-4", this "large - small - large" angle increment reflects the inadequacy of the control ability and the unreasonable changes in body posture of Zheng Wang in the process of rotation and acceleration.

\section{Acknowledgments}

This study was financially supported by hammer throw project of Zheng Wang team preparing for the Tokyo Olympic Games (2017) of China Track \& Field Team Integrated Technology Services Fund.

Project Number: 2017HT041 


\section{References}

[1] Borman , W .C .Motow idlo, S .J .Task performance and contex-tual performance:the meaning for personnel selection research, J .Sci.Hu man Performa nce. 12(1997)99 -109 .

[2] Organ , D.W .The motivational basis of organizational citizenship be-havior, J.Sci.Research in Organizationa l behavior .21(1990)43 -72 .

[3]McClelland , D .C .Testing for Competence rather than for Intelli-gence,J.Sci.Oxford :A merican Psychologist14 (1973)1 -4 .

[4] Panoutsakopoulos V, Papaiakovou G I, Katsikas F S, et al. 3D Biomechanical Analysis of the Preparation of the Long Jump Take-Off, J. 11(2010)55-68. 